\title{
Minimally invasive repair of pectus excavatum
}

\author{
André Hebra, Bennett W. Calder, Aaron Lesher
}

Division of Pediatric Surgery, Medical University of South Carolina, Charleston, SC 29425, USA

Correspondence to: André Hebra, MD. H. Biemann Othersen Professor of Surgery, Chief-of-Surgery, MUSC Children's Hospital, Division of Pediatric Surgery, Medical University of South Carolina, 96 Jonathan Lucas St, 417 CSB MSC 613, Charleston, SC 29425, USA. Email: hebra@musc.edu.

\begin{abstract}
Pectus excavatum, an acquired or congenital depression of the anterior chest wall, is the most commonly occurring chest wall deformity. Patients with pectus excavatum experience psychosocial and physiologic consequences such as impaired social development and pulmonary and/or cardiac dysfunction as a result of the deformity. Traditionally, repair of the defect was performed with a major open operation, the most common being based on modifications of the Ravitch procedure. In the late 1990's, the operative approach was challenged with a new minimally invasive technique described by Dr. Donald Nuss. This approach utilizes thoracoscopic visualization with small incisions and placement of a temporary metal bar positioned behind the sternum for support it while the costal cartilages remodel. Since introduction, the minimally invasive repair of pectus excavatum (MIRPE) has become accepted in many centers as the procedure of choice for repair of pectus excavatum. In experienced hands, the procedure has excellent outcomes, shorter procedural length, and outstanding cosmetic results. However, proper patient selection and attention to technical details are essential to achieve optimal outcomes and prevent significant complications. In the following, we describe our perspective on pectus excavatum deformities, operative planning, and technical details of the MIRPE procedure.
\end{abstract}

Keywords: Pectus excavatum; Nuss; minimally invasive repair of pectus excavatum (MIRPE); minimally invasive pectus repair; thoracoscopy; pectus bar; funnel chest; Ravitch

Received: 02 March 2016; Accepted: 22 March 2016; Published: 05 April 2016.

doi: $10.21037 /$ jovs.2016.03.21

View this article at: http://dx.doi.org/10.21037/jovs.2016.03.21

\section{Introduction}

Pectus excavatum is a congenital deformity of the anterior chest wall \& sternum believed to be a result of abnormal growth of the ribs and cartilage with consequent posterior displacement and angulation of the sternum. This causes the sternum to have a "caved-in" appearance. Pectus excavatum accounts for almost $90 \%$ of all congenital chest wall deformities and occurs in as many as 1 of every 300 to 400 live births (1-4). Male patients with pectus excavatum outnumber female patients at a ratio of 2:1 to $9: 1$ and the vast majority of patients are of Caucasian descent $(5,6)$. Several disorders and other malformations are associated with the pectus excavatum deformity including Marfan, Noonan, and Poland Syndromes and scoliosis. Though over $1 / 3$ of patients have a positive family history of pectus excavatum, no single gene has been identified as a causative factor of the deformity $(3,6,7)$.
Pectus excavatum is usually diagnosed shortly after birth with most cases identified by the second year of life (5). However, many patients do not come to medical attention until puberty or adolescence when the condition has a tendency to worsen and become much more noticeable. Figure 1 is a 3 -month old asymptomatic infant noted to have pectus excavatum and Figure 2 is a 17 -year-old male with a severe pectus excavatum that was considered to be very mild in infancy. The anatomic characteristics and physiologic significance of pectus deformities can vary widely. Patient presentations range from those with minor defects and cosmetic and/or psychological implications only to severe deformities with associated compromise of pulmonary \& cardiac function. As the appearance of the malformation may worsen with growth, symptoms associated with pectus excavatum may also become more apparent as patients age. Pre-teen or teenage patients often 


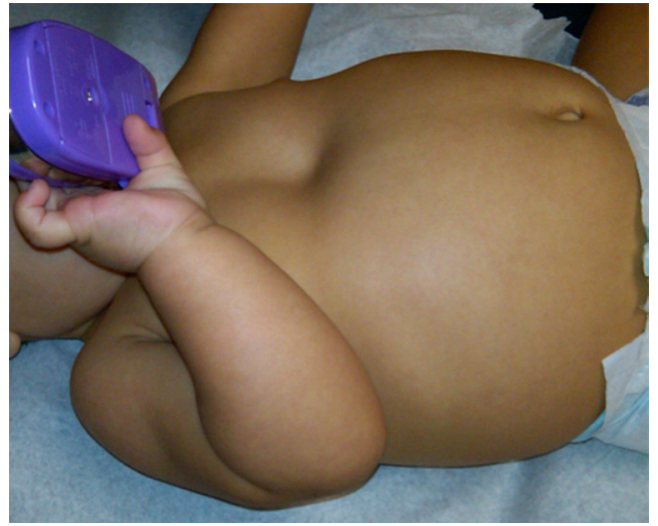

Figure 1 Image of an asymptomatic 3-month old infant with a significant pectus excavatum. The condition will typically worsen as the child gets older, becoming much more pronounced after puberty.

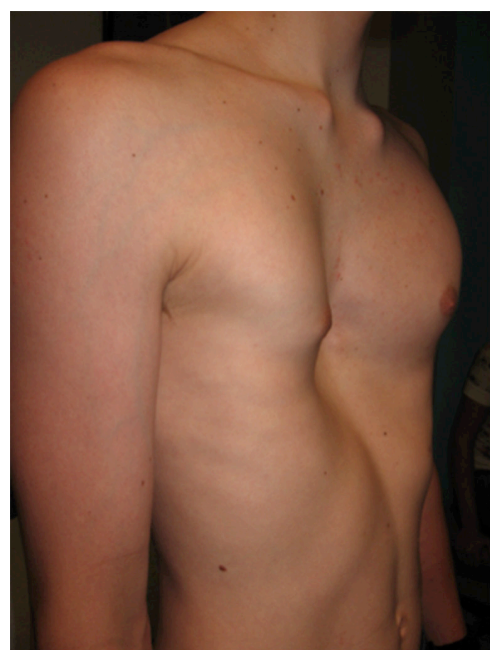

Figure 2 A 17-year-old male with severe pectus excavatum; preoperative Haller index of 6.5.

develop negative self-images and as a result experience impaired social development and depression. Patients may also increasingly report musculoskeletal complaints, such as back and chest pain, or cardiopulmonary complaints such as dyspnea on exertion and diminished exercise tolerance, which may be related to decreased pulmonary reserve, decreased forced vital capacity, decreased maximal voluntary ventilation, or limitation of cardiac stroke volume $(8,9)$. Furthermore, patients with significant pectus deformities may report palpitations related to mitral valve prolapse, arrhythmias, and atrial \& ventricular compression. Pectus excavatum and pectus carinatum are frequently associated with scoliosis (20-30\% of cases). Although such association

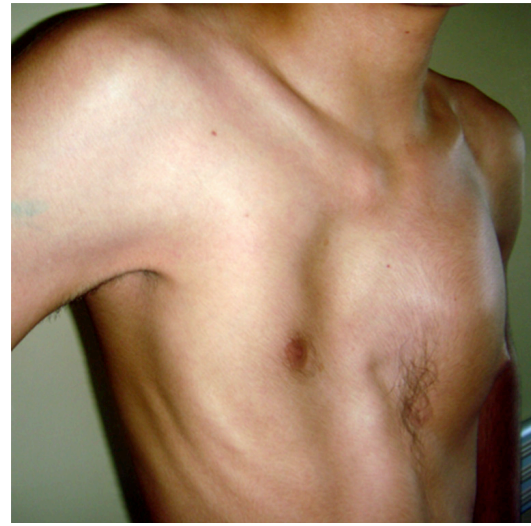

Figure 3 Adult patient with pectus excavatum and Poland's syndrome.

is probably coincidental, the poor posture noted in many patients with pectus deformities may be a key factor in the development of chest and back pain. The majority of pectus patients have a Marfanoid body habitus. A small number of such patients may have true Marfan or Poland syndrome. Figure 3 illustrates the appearance of an adult patient with pectus excavatum and Poland's syndrome in which the right pectoralis major muscle is absent. Figure 4 is a 10 -yearold female patient with true Marfan syndrome and a severe pectus excavatum with pre-operative Haller index of 12, as demonstrated on the computed tomography (CT) scan of the chest. Most Marfan's patients that have a pectus excavatum will have a very significant caved-in deformity of the chest.

In general, surgical repair of pectus excavatum is indicated for severe defects, functional impairment, and psychosocial factors. Traditionally, the operative correction of the deformity was performed utilizing the Ravitch technique, which involves the resection of abnormal rib cartilages with elevation of the sternum through an anterior transverse incision. In the 1980s-90s, Dr. Nuss performed his minimally invasive approach for repair of pectus excavatum in several young children as an alternative to the open procedure. During a MIRPE procedure, the sternum is elevated and secured in place with a temporary stainless steel prosthesis placed through small lateral incisions under thoracoscopic visualization. The first data on MIRPE outcomes was published in 1998 by Nuss and coauthors and published in the Fournal of Pediatric Surgery (10). The technique quickly gained popularity among surgeons and patients soon after. Since then, modifications to the procedure and increased surgeon experience have 

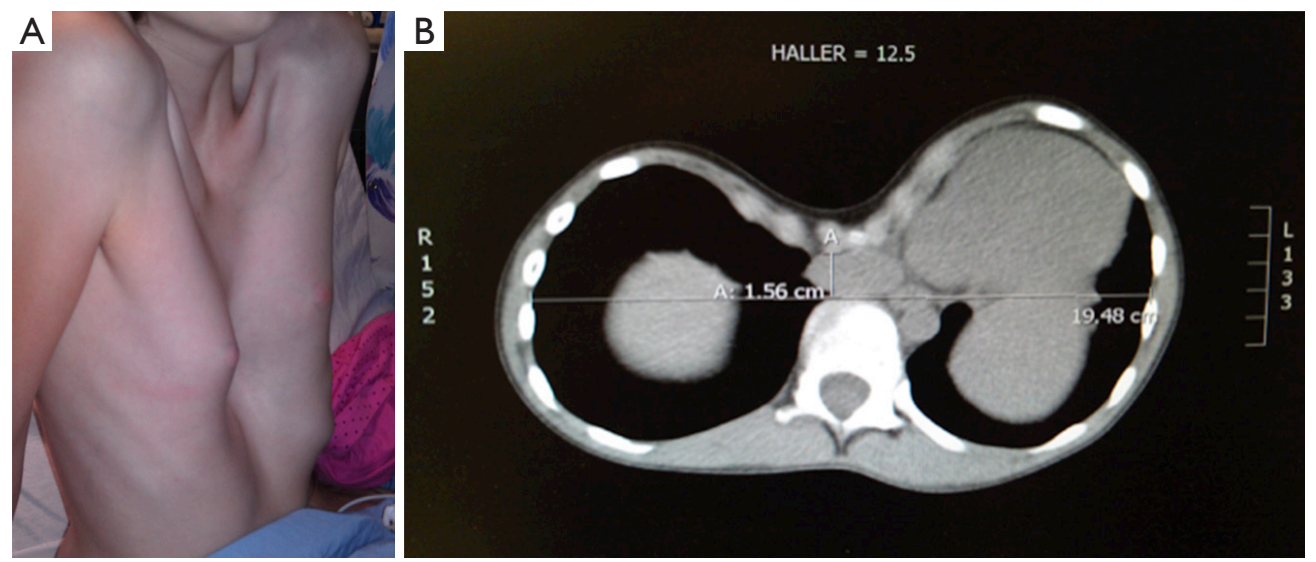

Figure 4 A 10-year-old female patient with true Marfan syndrome and a severe pectus excavatum with pre-operative Haller index of 12 , as demonstrated on the CT scan of the chest. (A) A 10-year-old female patient with Marfan's syndrome and severe pectus excavatum; (B) T scan of patient shown in (A). Haller index measured 12.5. Note the lower sternum touching the IVC and the asymmetry of the chest. CT, computed tomography.

contributed to excellent overall outcomes. However, as is the case with any invasive procedure, success is directly related to proper patient selection, careful patient preparation, and meticulous surgical technique.

\section{Patient selection and work-up}

Patients with pectus excavatum usually present in their pre-teen or early teenage years when accelerated growth accentuates the deformity. Evaluation for operative correction of the deformity consists of a complete history and physical, imaging, physiologic testing, and subspecialty consultation as indicated. Subjectively, patients may have complaints of non-cardiac chest pain, back pain, palpitations, shortness of breath with exertion, or decreased exercise tolerance. On exam, the severity of the deformity may range from mild depression to a deep irregular concavity extending almost to the spine. The patient may have "pectus posture" manifesting as forward slumping of the shoulders and a hunched over appearance. On auscultation, heart sounds are usually displaced leftward, the click of a prolapsing mitral valve might be apparent, and a flow murmur may be present. Breath sounds may be decreased at the bases. Asymmetry of the pectoralis muscle and chest wall/ribs should be noted pre-operatively, since there will be a persistent chest asymmetry despite successful MIRPE.

Imaging is an important component of the evaluation of a patient presenting with pectus excavatum and usually include non-contrast chest CT (on expiration). CT imaging is important to calculate the Haller index, which is derived by dividing the anterior-posterior diameter by the transverse diameter. It has been accepted that a Haller index of 3.2 indicates a severe deformity and is frequently considered an indication for surgery. Additionally, CT imaging provides anatomical information related to chest asymmetry which can be valuable to operative planning.

Echocardiography should be considered in all patients with pectus excavatum to evaluate for outflow obstruction related to the pectus and/or to evaluate for associated cardiac abnormalities. Electrocardiography is performed in cases with suspected cardiac dysrhythmia, which is rare. In order to demonstrate cardio-pulmonary impairment related to the pectus excavatum, an exercise stress test performed in the exercise physiologist's lab should be considered. It has been demonstrated that patients with a Haller index $>4.5$ will usually have significant exercise intolerance with a restrictive pattern on preoperative pulmonary function tests (9).

As a rule at our institution, the pre-operative workup of patients with pectus excavatum includes a non-contrast limited CT scan of the chest for measurement of the Haller index and for determination of the degree of chest asymmetry and heart displacement (as illustrated in Figure 4). An echocardiogram to determine the degree of cardiac compression. The echo may demonstrate changes in stroke volume and even mitral and/or tricuspid valve regurgitation. Stress exercise test is also obtained and it usually demonstrates decrease in stroke volume and exercise tolerance. Again, the severity of the pectus will determine the degree of physiologic impairment. Figure 5 


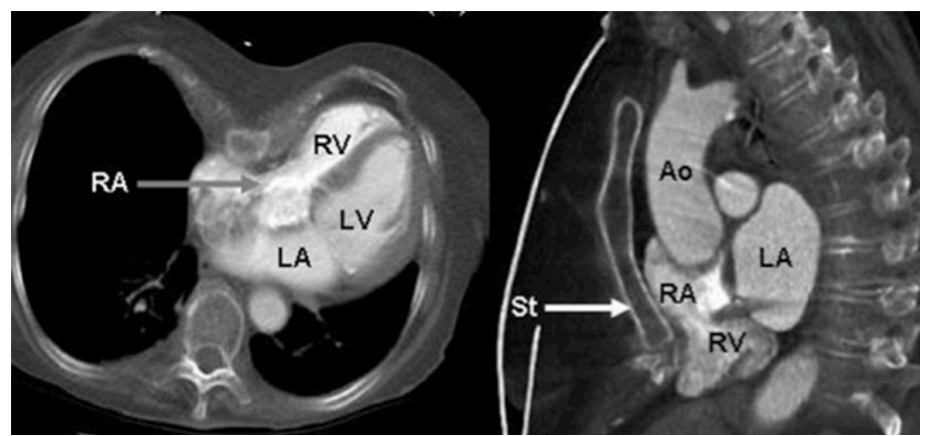

Figure $5 \mathrm{MRI}$ of the chest illustrating right atrial compression caused by the pectus excavatum. The degree of compression is variable and depends on the severity of the pectus deformity.
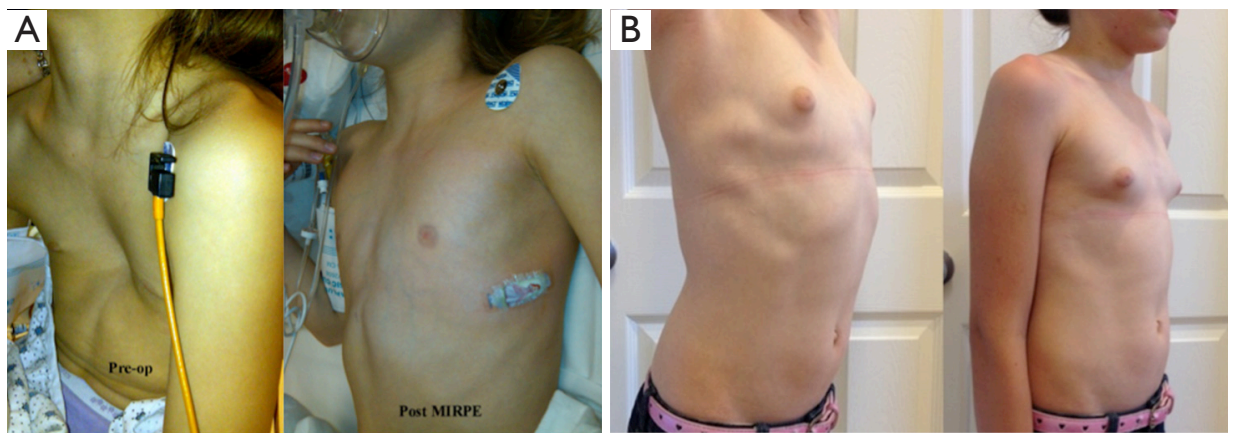

Figure 6 An 8-year-old child with severe pectus excavatum. (A) Pre- and post-operative images of an 8-year-old female with pectus excavatum (Haller index of 5.3). Note the excellent correction immediately after MIRPE; (B) same patient as in (A), now 3 years after repair. Excellent sustained correction of the pectus. The small lateral chest wall incisions are almost invisible. MIRPE, minimally invasive repair of pectus excavatum.

demonstrates a significant degree of atrial compression seen on a chest MRI caused by the pectus excavatum.

Indications for surgery include severe deformity (Haller Index 3.2 or greater), exercise limitations with exertional dyspnea, chest pain, documented decreased pulmonary function, abnormal exercise tolerance testing, symptomatic cardiac compression, and need for future sternotomy. In addition, significant psychosocial and body-image perception problems should be taken into account when determining the need for surgery. In the United States, many insurance companies require pre-operative testing to include measurement of Haller index by CT scan, pulmonary function testing, and echocardiography. Timing of the operation is also an important consideration as the ideal candidates for repair are typically between 8 and 14 years of age. The flexible chest walls of pre-pubertal patients are better suited to reshaping with the pectus bar. Moreover, the pain after repair is less when compared to the stiffer chest wall of older patients (teenagers and adults).
It is usually not recommended to perform the procedure in children younger than 7 years of age as recurrence of the pectus may become a problem in the future. In addition, post-procedure participation with respiratory and physical therapy may be challenging for young patients. Figure 6 is an example of an 8-year-old child with severe pectus excavatum. The photographs show the pre and immediate post-operative images (Figure $6 A$ ) as well as images 3 years after the repair and post bar removal (Figure $6 B$ ). Relative contraindications for repair include the presence of significant primary cardiac dysfunction (not related to the pectus), neurodevelopmental delay, concurrent complex congenital abnormalities, and immunosuppression.

\section{Pre-operative preparation}

Once indications for repair have been established, preoperative preparation can begin. Attention to patient and caregiver education, as well as the process of informed 


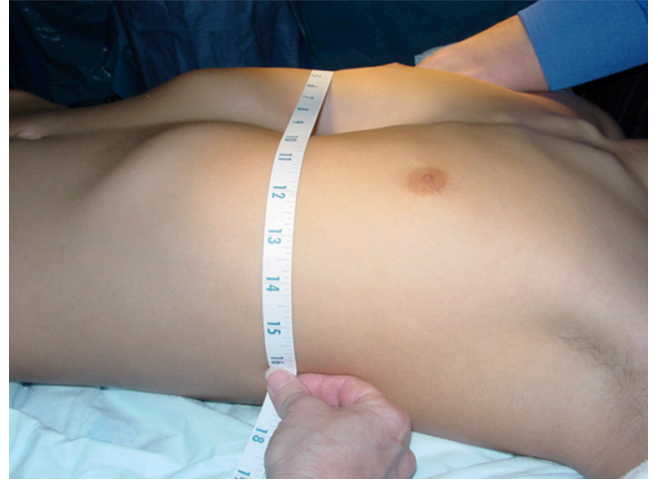

Figure 7 Measurement of the chest at the time of surgery. This will determine the size of the pectus bar necessary for the correction of pectus excavatum.

consent, is essential to successful outcomes and patient satisfaction. Elements of the informed consent should include a discussion of therapeutic options: open repair, minimally invasive, and non-operative treatments. The risk of complications including bar displacement, need for reoperation, and the unlikely but real possibility of cardiac, vascular, and pulmonary injury should be carefully discussed with the patient and his/her family. Expectations of the patient should be addressed and the potential for chest asymmetry to persist after repair discussed, particularly in cases with chest asymmetry, rib flaring, and sternal rotation. Details of post-operative pain management and expectations of patient participation with therapy should be explained in appropriate detail.

Patients with indications for repair who wish to proceed with surgery should be measured from mid-axillary line to mid-axillary line to estimate the pectus bar length. Bar length is typically $1-2 \mathrm{~cm}$ less than this length. If the patient has an allergy to certain metal alloys, a titanium bar should be pre-ordered for the procedure. In our practice, specific questions regarding a history of metal hypersensitivity should be asked of parents and patient, including reactions to belt buckles, wristwatches, or jewelry. Patients are to be NPO 12 hours prior to general anesthesia.

\section{Equipment preference card (for MIRPE)}

Open and thoracoscopic instruments should be available for the procedure. A $5 \mathrm{~mm}$ thoracoscopic/laparoscopic set of instruments with a $5 \mathrm{~mm} 30$ degree laparoscope; Pectus bars (various sizes); Zimmer bar bender; Pectus bar flipper instrument. A typical instrumentation list is outlined here:
- Drape: PEDS lap cholecystectomy pack;

- Cloth towels $\times 4$;

- Ioban (cut two strips 2" wide);

- Prep: chloraprep $25 \mathrm{~mL}$ application $\times 2$;

Instruments:

- PEDS major and PEDS pectus tray;

- PEDS pectus accessory tray;

- PEDS 5 mm lap lenses 30 degree set;

- HD camera for thoracoscopy-use storz (or equivalent) non-disposable trocar;

- Synthes tabletop plate bender;

- Sterile twill tape $\times 2$ (open 1) (peel pack);

- Sterile paper measure (peel pack);

- Cloth gown.

Supplies:

- Pyxis or 5;

- Half sheet;

- Suction tubing;

- Bovie ×2;

- 18 GA spinal needle;

- Grounding pad-weight specific $\times 2$.

Have available:

- Kittners;

- Q-tips;

- 14 Fr Robnel catheter;

- Sterile water 1,000 cc bottle;

- Note: will insert Foley on all pts with epidural;

- Two bovies-need extra bovie machine;

- Position: supine, arms extended \& out-two arm boards with padding.

Sutures:

- 3-0 Vicryl SH $\times 2$;

- 5-0 Monocryl p-3 ×3;

- 1 Prolene CTX $\times 1$;

- 1 PDS CTX $\times 3$.

- \#2 surgical steel LS-1

- Mastisol*, $1 / 2$ " steri strips*, bandaids

- Vacuum bells (various sizes).

\section{Procedure}

The first step is to determine the estimated length of the pectus bar to be used for the repair. This can be accomplished by measuring the distance between right and left mid-axillary lines (Figure 7). Most surgeons prefer to use a bar that is shorter than that measured distance by 1-2 inches. Two small $2 \mathrm{~cm}$ skin incisions are made in the lateral right/left chest in the mid-axillary line bilaterally 


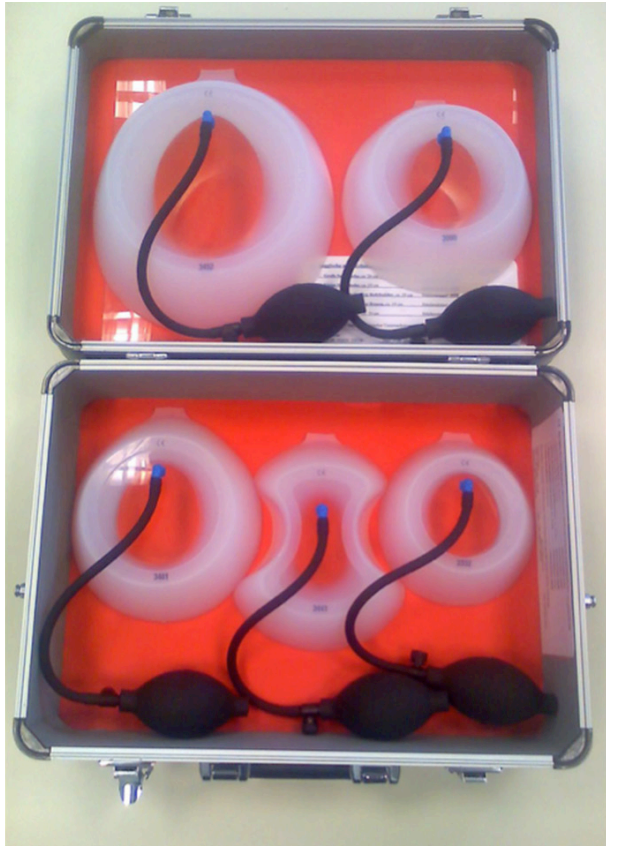

Figure 8 The Eckart Klobe vacuum bells (complete set)-this device can be used intra-operatively to elevate the sternum during placement of the pectus bar.

that correspond to the marks used to measure bar length and should be in line with the deepest point of the concave deformity. Skin flaps are raised anteriorly from both incisions to the top of the pectus ridge at the level of the mid-clavicular line. This pocket is extended posteriorly to allow for the bar and stabilizer to sit comfortably underneath the skin. A $5 \mathrm{~mm}$ blunt trocar is placed in the right lower chest, two intercostal spaces below the level of the lateral chest wall incision on the right side. A $5 \mathrm{~mm}$ 30 degree thoracoscope is then introduced to allow for guidance and visualization when creating the substernal dissection in the anterior mediastinal space. A pectus bar introducer instrument is used and three sizes are available (short, long, and extra-long).

The pectus introducer is inserted through the right lateral incision and the tip is used to dissect through the intercostal muscles in the intercostal space corresponding to the area of maximal sternal depression. The introducer is advanced across the anterior mediastinal space immediately under the sternum under careful thoracoscopic guidance. Great care should be taken during this step of the procedure as the pericardium is teased from the undersurface of the sternum. It is usually recommended to attempt to raise

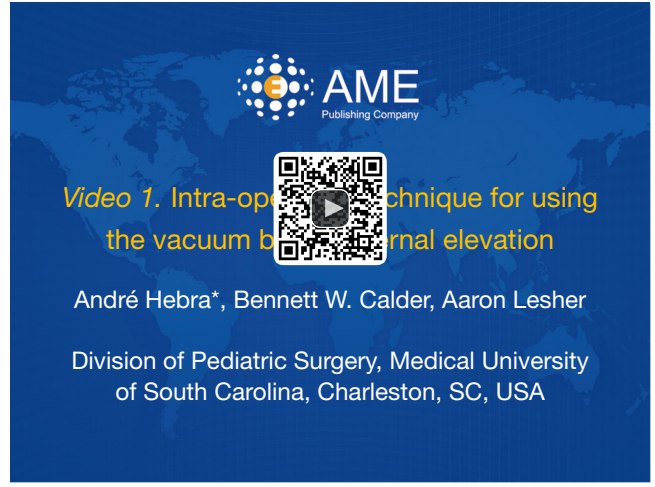

Figure 9 Intra-operative technique for using the vacuum bell for sternal elevation (11). By applying negative pressure, the sternum is raised anteriorly which facilitates passage of the pectus bar across the anterior mediastinal space. Sternal elevation is important during MIRPE in order to minimize the risk of injury to the heart or pericardium. MIRPE, minimally invasive repair of pectus excavatum.

Available online: http://www.asvide.com/articles/951

the sternum during this part of the procedure. This can be accomplished with the use of a vacuum bell (Figures 8,9), Rultract retractor, and/or Park crane technique (12). Of note, the vacuum bell has also been used as a treatment alternative for non-operative management of mild cases of pectus excavatum. During MIRPE, the introducer is passed to the contralateral side with the sternum lifted anteriorly under careful thoracoscopic visualization and guidance. The tip of the introducer is pointed anteriorly towards the sternum and chest wall at all times. The pericardium is gently pushed posteriorly. The tip of the introducer is then brought out through the corresponding intercostal space on the left side. Then, using the introducer as a guide, umbilical or cloth tape is pulled through the newly dissected tunnel and it will serve to guide the placement of the pectus bar across the anterior mediastinal space. Typically the bar is bent by the operating surgeon at the time of the repair. This can be accomplished using hand-held bar benders or using a bar bending table instrument (Figure 10). The bar should be given a smooth curvature to conform to the patient's chest and to provide optimal displacement of the sternum anteriorly with correction of the caved-in sternal deformity. Insertion of the bar into the chest through the lateral chest wall incision and through the intercostal space corresponding to the maximal depression of the sternum (at the mid-clavicular line) is done with the convexity facing 
posteriorly, the reverse of the final position. Then, using a pectus bar flipper instrument, the bar is turned over 180 degrees and the ends placed under the subcutaneous tissues. Figure 11 is an intra-operative view of the bar in place behind the sternum before and after flipping it 180 degrees. One can see the mediastinal structures once the sternum is fully elevated and supported by the pectus bar. It is important to place the bar ends anterior to the muscle fascia and avoid placement of the bar underneath fascia or within the muscle. Placement and shaping of the bar can be adjusted as needed to improve the repair. This may require removal and replacement of the bar or it may be accomplished using hand held bar-benders. Of note, it

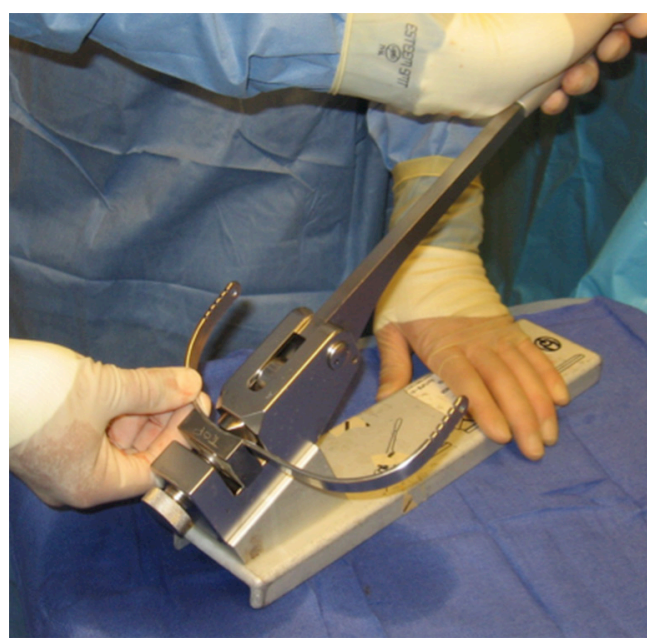

Figure 10 Bar bender device utilized for shaping the pectus bar at the time of surgery. The bar is given a smooth curve to conform to the patient's chest in order to provide maximal correction of the caved-in sternal deformity. has become increasingly common to place more than one pectus bar, particularly in older patients. The placement of two bars may improve the appearance of the chest and it may also minimize the risk of bar displacement after surgery. Figure 12 is a chest $\mathrm{X}$-ray of a patient with two pectus bars. Once the operating surgeon is satisfied with bar position and appearance of the chest, the need for bar stabilization bars is assessed. If lateral stabilizing bars are necessary to minimize the risk of rotation of the pectus bar, stabilizers can be placed on one or both sides and secured to the pectus bar with \#1 stainless steel wire suture. Most surgeons try to place stabilizers in only one side of the pectus bar. The pectus bar and stabilizers are then secured against the muscle fascia with non-absorbable \#1 Prolene sutures and \#1 PDS absorbable sutures on the contra-lateral side that does not have the stabilizer. The use of permanent sutures to secure the bar on only one side allows for reopening only one incision at the time of pectus bar removal. Additionally, a third point of fixation is recommended by the authors to prevent bar displacement (13). This is accomplished using absorbable \#1 PDS sutures placed around a rib and around the pectus bar, lateral to the sternum (Figure 13). One or more of such sutures can be placed in order to maximize bar stability. The use of thoracoscopy and Tuohy spinal needle allows for precise placement of such sutures without risk of cardiac, vascular or pulmonary injury.

The soft tissues are then closed in layers and the skin closed with a subcuticular suture. To ensure evacuation of any residual air within the pleural cavity, a temporary red rubber catheter threaded through the trocar site is connected to suction and Valsalva maneuver is performed. Post-operative chest radiograph is routinely obtained by

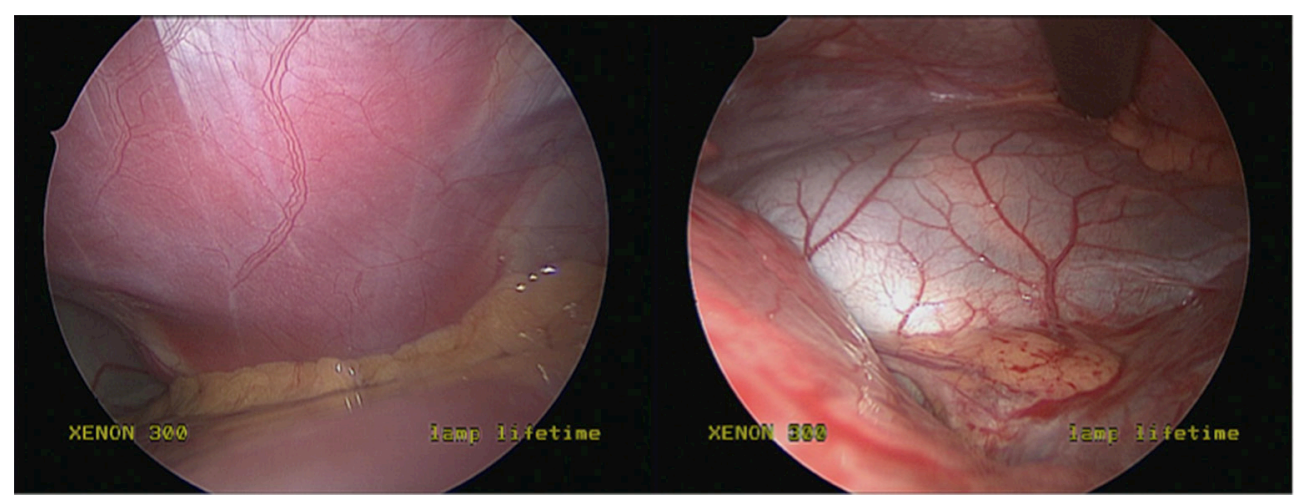

Figure 11 Intra-operative image of the thoracoscopic view (from the right chest) of a pectus excavatum before and after placement of the pectus bar. A single pectus bar provided complete correction of the caved-in sternum and the mediastinum returned to normal position. 
our group in order to ensure that full lung expansion has occurred and that the patient does not have a significant residual pneumothorax.

\section{Role of team members}

It is important to have a skilled assistant when performing MIRPE. This will facilitate safe placement of the pectus bar and will improve operating room efficiency as both (surgeon and assistant) can work simultaneously on each side of the chest during the key portions of the operation. Another important member of the team is the anesthesiologist \& pain management expert. Many centers prefer the use of combined general anesthesia and thoracic epidural for management of pain during and after the procedure. A discussion related to pain management will follow in the next session. Good communication and collaboration with the anesthesiologist is essential not only for the intra-op

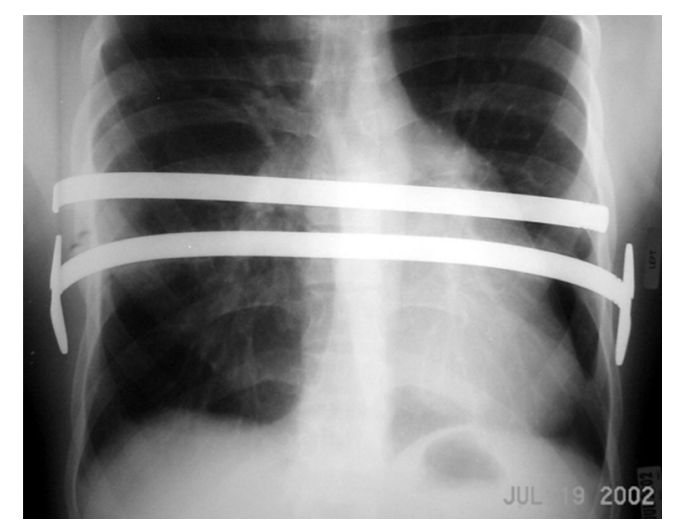

Figure 12 Chest radiograph of an adult patient treated for pectus excavatum with placement of two pectus bars. Stabilizers were necessary only on the lower bar. but also for post-operative management. Consultation with physical therapy is initiated on post-operative day one in order to help patients initiate ambulation, set expectations, and to develop a post-operative treatment plan related to the patient's activity level. In many cases the physical therapist remains involved during the first eight weeks of post-operative recovery.

\section{Post-operative management}

Pain management is especially important after repair of pectus excavatum. At our institution we developed a multi-modal approach incorporating epidural analgesia, patient controlled narcotic analgesia, and non-steroidal anti-inflammatory drugs. In our experience this strategy is important for patient tolerance of pulmonary toilet maneuvers, good posture, and physical therapy. However, it is important to note that peril-operative pain control regimens for MIRPE patients, particularly relating to the use of thoracic epidural analgesia, are an area of debate. Thoracic epidural analgesia has historically been a major component of post-operative pain management in patients undergoing repair of chest wall deformities. However, more recently the practice of routine thoracic epidural use in MIRPE patients has been questioned. In 2012 a prospective randomized trial comparing epidural to patient controlled analgesia for postoperative pain found that thoracic epidural use was associated with higher costs, no significant difference in length of hospital stay, and subjective pain ratings that were lower only on post-operative days zero and one (14). A 2014 meta-analysis comparing epidural and patient controlled analgesia concluded that although epidural is associated with lower pain scores in the immediate post-operative period, there was no clinical
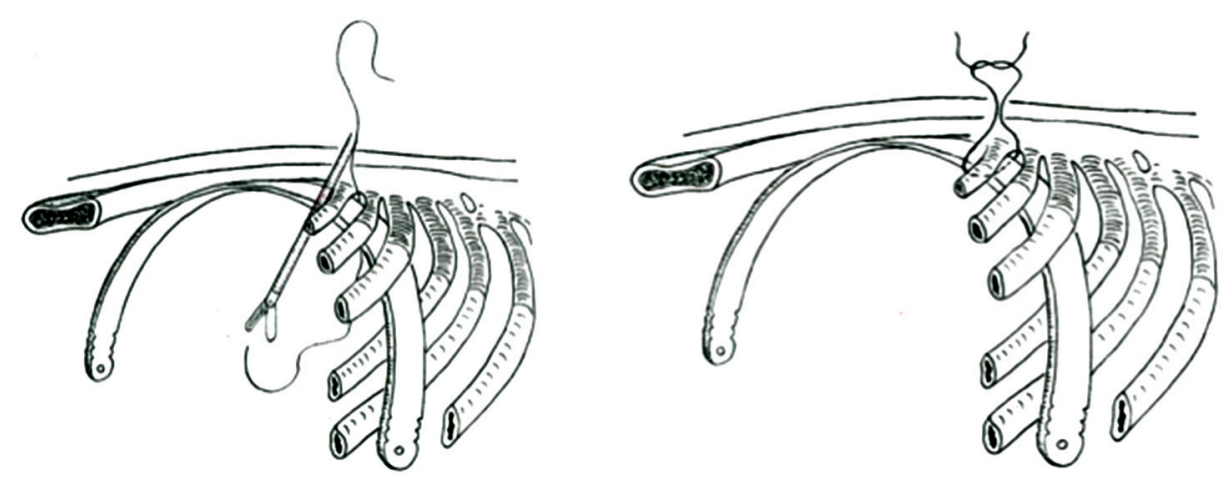

Figure 13 Illustration of the technique for placement of the "third point of fixation" (or peri-costal sutures) for stabilization of the pectus bar. This is an effective method for minimizing the risk of bar displacement. 


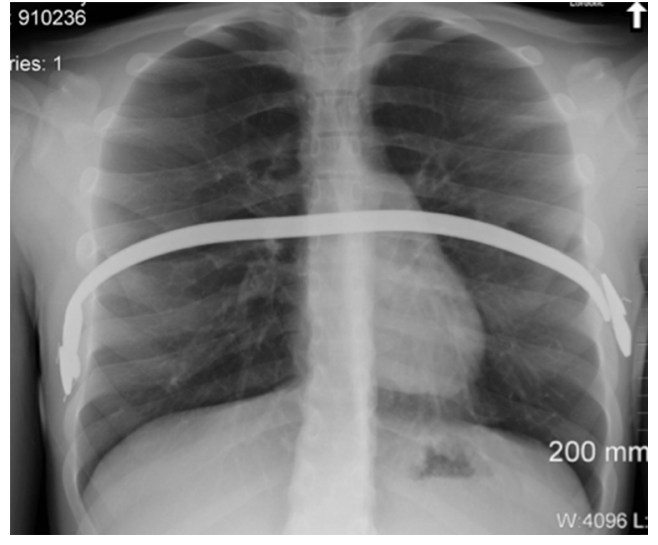

Figure 14 Chest radiograph of a patient that experienced superior displacement of the pectus bar with separation of the lateral stabilizers from the bar. This patient required re-operation for correction of this problem.

difference overall in post-operative outcomes (15). We continue to utilize epidural analgesia with excellent outcomes and consider it an important component of postoperative pain management especially in the immediate post-operative period and in older patients with less flexible chest walls. The decision to include epidural analgesia as a component of post-operative pain management should be made on a case-by-case basis with careful discussion of the risks and benefits with the patient or guardians. After the epidural catheter is removed, if one was placed, and the patient successfully transitioned from intravenous to oral narcotic medication with good pain control, the patient may be discharged, usually on day 4-7. Heavy lifting is restricted for 1 month. After 30 days the patient should participate in a physical activity program that will speed healing and promote remodeling of the chest wall. No contact sports are permitted for a period of 6 months. Bar removal is done as an outpatient under general anesthesia on average 3 years after bar placement.

The spectrum of adverse outcomes related to the minimally invasive technique is somewhat variable, and most complications today are considered rare and unusual (16-21). Preliminary reports when the operation was considered 'new' suggested a higher rate of complications, with the most commonly reported complication being related to pectus bar displacement $(16,17)$. Figure 14 is a chest radiograph of a patient that experienced pectus bar displacement and separation of the stabilizers from the pectus bar. The following is a list of reported complications after MIRPE (and the estimated incidence for each):

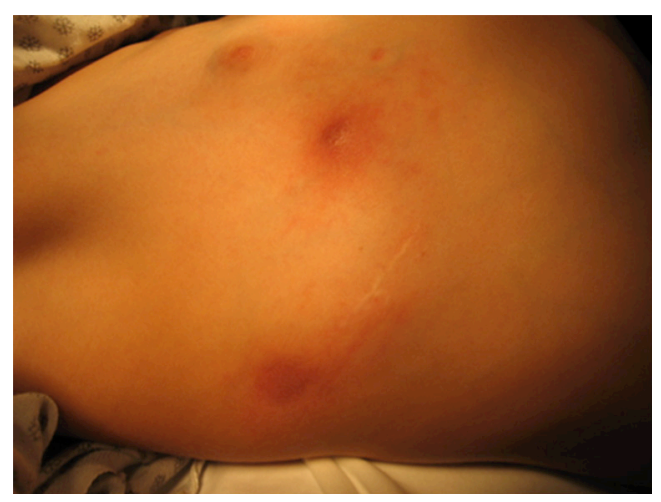

Figure 15 Skin rash surrounding the right lateral chest wall incision two months after MIRPE. The patient was diagnosed with metal allergy as the cause of the skin reaction. MIRPE, minimally invasive repair of pectus excavatum.

- Pectus bar displacement-requiring reoperation $(2.5 \%)$

- Pneumothorax-requiring chest tube (3\%);

- Overcorrection (3\%);

- Epidural catheter related complications (4\%);

- Bar allergy (1-2\%);

- Wound infection (1\%);

- Pleural effusion (1\%);

- Thoracic outlet syndrome $(<0.5 \%)$;

- Pericarditis $(<0.5 \%)$;

- Cardiac injury $(<0.5 \%)$;

- Sternal erosion $(<0.5 \%)$;

- Death $(<0.1 \%)$.

Of note, the overall morbidity of the procedure was initially reported to be high, at almost $20 \%$, with the most common complication being bar displacement or rotation requiring reoperation (17). However, with improvements and modifications to the technique, complication rates have dropped by over $75 \%$ (22). Pre-operative screening for metal allergy has resulted in a decreased incidence skin rash and systemic reactions related to this unusual problem. Figure 15 is a representation of a young patient that developed a significant skin rash around the surgical incision site related to allergy to metal alloys found in the pectus bar. Fortunately this problem will rarely require removal of the pectus bar. If metal allergy is identified preoperatively, the patients should receive a Titanium bar for the correction of the pectus.

A recent analysis of Pediatric NSQIP data reported overall morbidity of MIRPE to be $3.8 \%$ with no mortalities 


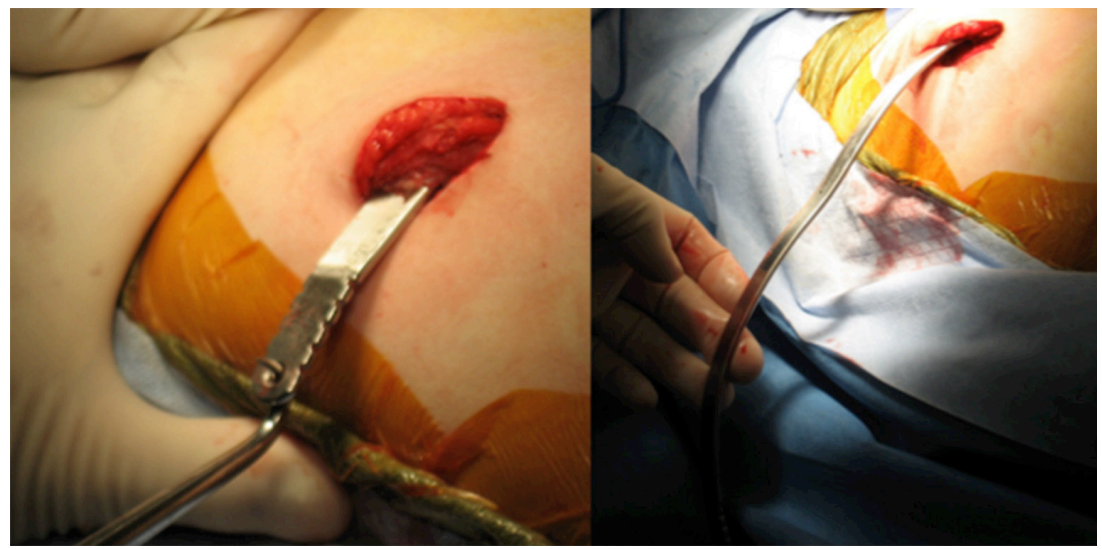

Figure 16 Technique for pectus bar removal. The old incision site is re-opened and the bar is removed by applying traction towards the floor. The bar should not be pulled straight across the chest as this may cause injury to the mediastinum.

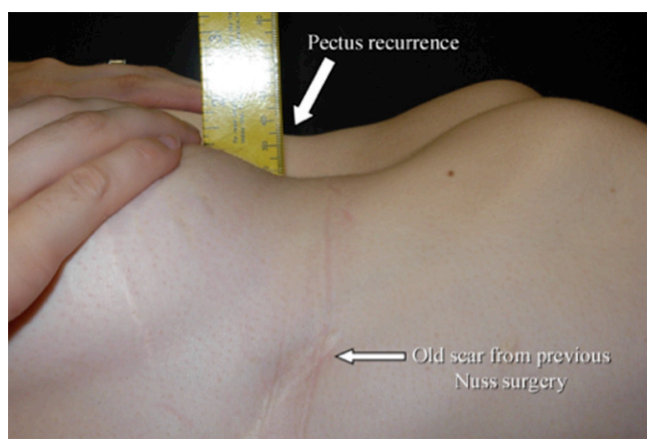

Figure 17 Image of a 17-year-old patient with recurrent pectus excavatum post minimally invasive repair initially performed at 6 years of age.

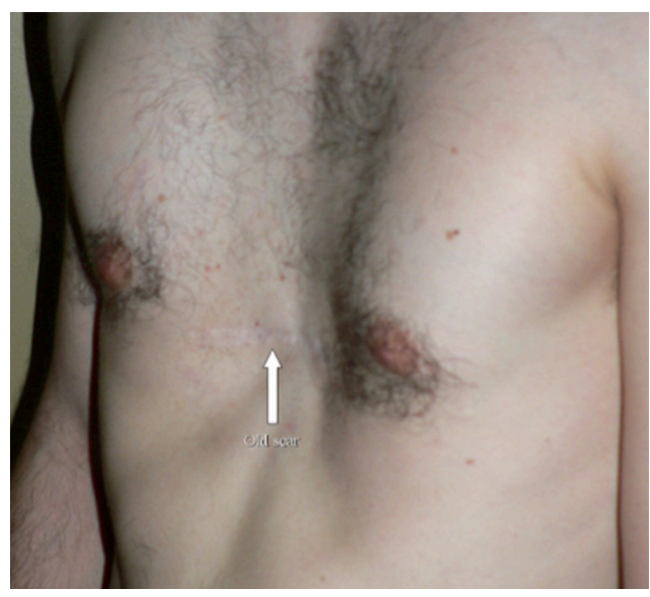

Figure 18 Adult patient with a recurrent pectus excavatum post open modified Ravitch repair performed initially performed at 14 years of age. identified in their dataset (22). Though complications after MIRPE are fairly rare today, it is important to recognize the impact of the procedural learning curve on declining rates and be aware that these data come from centers with extensive experience in the repair of chest wall deformities. Furthermore, though rare, mortality during or after the procedure has been reported (23). Given that pectus excavatum is a condition that rarely causes any life-threatening problems, it is especially important to educate patients and families on the risks associated with the operation, both bar insertion and removal, through discussion and patient education materials in order to allow all parties to make a calculated, well-informed decision about surgical treatment.

Pectus bar removal surgery can usually be accomplished as an outpatient procedure, 3 years after the primary repair. Re-opening of the lateral chest incisions and mobilization/ removal of the bar and stabilizer can be difficult if the patient has developed scar \& calcifications around the bar. A traction technique is applied for pectus bar removal as illustrated in Figure 16. It is important to pull the bar in such a way to conform to the patient's chest in order to minimize risk of injury to mediastinal structures. The bar is pulled towards the floor and it can be unbent in the process using hand-held bar benders.

Pectus recurrence is considered very rare (less than $1 \%$ ) if the pectus bar has been left in place for 3 years or more. Figure 17 is an illustration of a patient that experienced pectus recurrence after a Nuss procedure performed at a young age. Figure 18 shows an adult patient being considered for minimally invasive pectus repair after a 


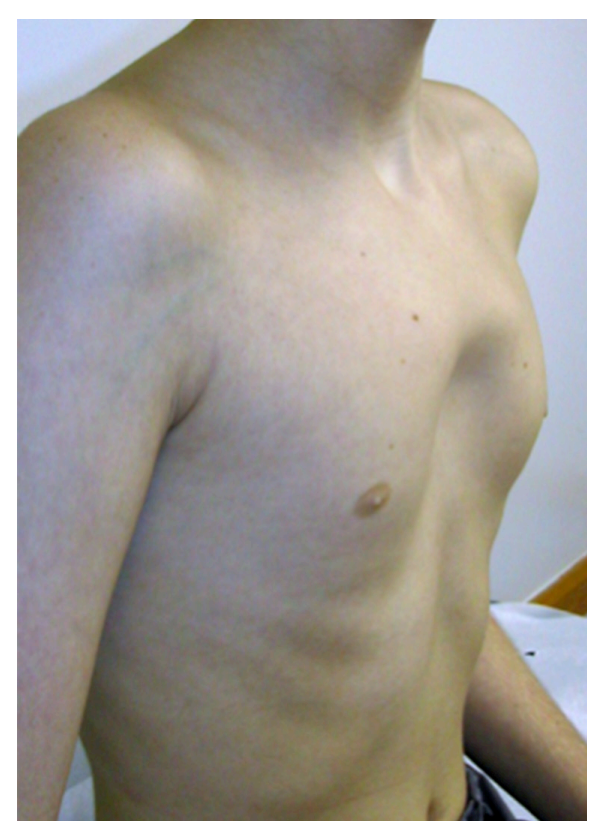

Figure 19 A 12-year-old child with pouter pigeon chest, also known as 'Horns of Steer' pectus deformity. Note the protrusion in the upper sternum and the caved in lower sternum.

recurrence following an open modified Ravitch operation performed early in life. Such cases can be challenging due to the presence of mediastinal adhesions from his previous surgery. It is usually accepted that the morbidity and mortality is higher in re-operative cases.

\section{Tips, tricks, and pitfalls}

In order to provide the best possible care to patients with pectus excavatum, it is important for surgeons to obtain adequate training in performing the minimally invasive procedure. The learning curve can be steep as the patient's presentation and type of pectus deformity (with or without asymmetry) vary greatly. In addition, the patient's age plays a significant factor as the operation is considered relatively easy in children less than 12 years of age and somewhat more complicated in teenagers and young adults. Literature review has demonstrated that the incidence of severe complications is more common in older patients.

The first step in optimizing outcomes is appropriate patient selection. Best outcomes are typically obtained in younger patients with a symmetric pectus excavatum. Such cases can be corrected with a single pectus bar and with minimal morbidity. Older patients or patients with significant chest asymmetry may require more than one bar and the shape of the bar must be appropriately customized in order to provide the best possible correction of the deformity. Patients with mixed \& complex deformities such as Pouter Pigeon chest may require a hybrid procedure (Nuss and open approach) in order to achieve an adequate cosmetic correction of the chest wall malformation. Figure 19 illustrates a case of a young patient with a Pouter Pigeon chest wall deformity. As previously mentioned, patients with previous chest procedures, including open heart surgery and Ravitch repair of pectus, are considered to be at higher risk for lifethreatening complications due to the likelihood of intrathoracic adhesions.

One of the critical steps of the minimally invasive operation is the insertion of the pectus bar passer across the anterior mediastinal space and the placement of the pectus bar. This can be challenging, particularly in older patients with a severe pectus deformity. Many experienced surgeons advocate the use of "sternal elevation techniques" for this part of the operation. This would include the intraoperative use of the vacuum bell device or the Rultract retractor. Figure 9 illustrates the intra-operative use of the vacuum bell for elevating the sternum during MIRPE. It is known that obtaining a critical view of the anterior mediastinal space during passage of the pectus bar is essential in order to minimize the risk of major problems related to cardiac, lung, or vascular injury. The second critical step of the operation is determining if more than one bar is necessary for the repair. In recent years, most surgeons have adopted the use of two or even three bars for the correction of the caved in deformity of the sternum. It seems that more than one bar aids in providing better stability to the bar and it also improves the cosmetic correction of the pectus. This is particularly true in older teenagers and young adults that have less pliable chest walls. The third critical step is assuring that the pectus bar is properly stabilized in order to prevent the occurrence of bar displacement. This would include the use of lateral stabilizers and third-point of fixation technique in which the bar is secured with peri-costal sutures (around the bar and around a rib). Bar displacement was reported as the most common complication after surgery in the early 2000's; today, bar displacement requiring re-operation is considered rare and reported in less than $2.5 \%$ of cases when proper bar fixation techniques are employed (24).

Controversy still exists as to the best approach for repair of pectus deformities: should it be an open or minimally invasive approach? There is no doubt that both operations are effective and considered safe. Surgeon and patient 
preference play a major role in determining which approach is best. Given the cosmetic advantage of the minimally invasive approach, the demand for the minimally invasive surgery for correction of pectus excavatum has grown in an exponential manner and it is likely that it will continue to do so. Prospective randomized trials comparing the two operations are not likely to be possible at this point in time given the significant amount of bias involved and the lack of major funding to conduct a study of that magnitude (25). Regardless of surgical approach used for the repair of pectus, it is important that surgeon and patient have realistic goals and understand the risks and benefits involved. Surgeon experience and patient selection are important in order to minimize the risk of adverse outcomes.

\section{Acknowledgements}

None.

\section{Footnote}

Conflicts of Interest: The authors have no conflicts of interest to declare.

\section{References}

1. Molik KA, Engum SA, Rescorla FJ, et al. Pectus excavatum repair: experience with standard and minimal invasive techniques. J Pediatr Surg 2001;36:324-8.

2. Coskun ZK, Turgut HB, Demirsoy S, et al. The prevalence and effects of Pectus Excavatum and Pectus Carinatum on the respiratory function in children between 7-14 years old. Indian J Pediatr 2010;77:1017-9.

3. Cobben JM, Oostra RJ, van Dijk FS. Pectus excavatum and carinatum. Eur J Med Genet 2014;57:414-7.

4. Chung CS, Myrianthopoulos NC. Factors affecting risks of congenital malformations. I. Analysis of epidemiologic factors in congenital malformations. Report from the Collaborative Perinatal Project. Birth Defects Orig Artic Ser 1975;11:1-22.

5. Brochhausen C, Turial S, Müller FK, et al. Pectus excavatum: history, hypotheses and treatment options. Interact Cardiovasc Thorac Surg 2012;14:801-6.

6. Koumbourlis AC. Pectus deformities and their impact on pulmonary physiology.Paediatr Respir Rev 2015;16:18-24.

7. Fonkalsrud EW, Dunn JC, Atkinson JB. Repair of pectus excavatum deformities: 30 years of experience with 375 patients. Ann Surg 2000;231:443-8.
8. Kelly RE Jr, Cash TF, Shamberger RC, et al. Surgical repair of pectus excavatum markedly improves body image and perceived ability for physical activity: multicenter study. Pediatrics 2008;122:1218-22.

9. Kelly RE Jr, Mellins RB, Shamberger RC, et al. Multicenter study of pectus excavatum, final report: complications, static/exercise pulmonary function, and anatomic outcomes. J Am Coll Surg 2013;217:1080-9.

10. Nuss D, Kelly RE Jr, Croitoru DP, et al. A 10-year review of a minimally invasive technique for the correction of pectus excavatum. J Pediatr Surg 1998;33:545-52.

11. Hebra A, Calder BW, Lesher A. Intra-operative technique for using the vacuum bell for sternal elevation. Asvide 2016;3:195. Available online: http://www.asvide.com/ articles/951

12. Park HJ, Jeong JY, Jo WM, et al. Minimally invasive repair of pectus excavatum: a novel morphology-tailored, patient-specific approach. J Thorac Cardiovasc Surg 2010;139:379-86.

13. Hebra A, Gauderer MW, Tagge EP, et al. A simple technique for preventing bar displacement with the Nuss repair of pectus excavatum. J Pediatr Surg 2001;36:1266-8.

14. St Peter SD, Weesner KA, Weissend EE, et al. Epidural vs patient-controlled analgesia for postoperative pain after pectus excavatum repair: a prospective, randomized trial. J Pediatr Surg 2012;47:148-53.

15. Stroud AM, Tulanont DD, Coates TE, et al. Epidural analgesia versus intravenous patient-controlled analgesia following minimally invasive pectus excavatum repair: a systematic review and meta-analysis. J Pediatr Surg 2014;49:798-806.

16. Engum S, Rescorla F, West K, et al. Is the grass greener? Early results of the Nuss procedure. J Pediatr Surg 2000;35:246-51; discussion 257-8.

17. Hebra A, Swoveland B, Egbert M, et al. Outcome analysis of minimally invasive repair of pectus excavatum: review of 251 cases. J Pediatr Surg 2000;35:252-7; discussion 257-8.

18. Marusch F, Gastinger I. Life-threatening complication of the Nuss-procedure for funnel chest. A case report]. Zentralbl Chir 2003;128:981-4.

19. Rushing GD, Goretsky MJ, Gustin T, et al. When it is not an infection: metal allergy after the Nuss procedure for repair of pectus excavatum. J Pediatr Surg 2007;42:93-7.

20. Shin S, Goretsky MJ, Kelly RE Jr, et al. Infectious complications after the Nuss repair in a series of 863 patients. J Pediatr Surg 2007;42:87-92.

21. Hebra A. Minimally invasive pectus surgery. Chest Surg Clin N Am 2000;10:329-39, vii. 
22. Sacco-Casamassima MG, Goldstein SD, Gause CD, et al. Minimally invasive repair of pectus excavatum: analyzing contemporary practice in 50 ACS NSQIP-pediatric institutions. Pediatr Surg Int 2015;31:493-9.

23. Bouchard S, Hong AR, Gilchrist BF, et al. Catastrophic cardiac injuries encountered during the minimally invasive repair of pectus excavatum. Semin Pediatr Surg

doi: 10.21037/jovs.2016.03.21

Cite this article as: Hebra A, Calder BW, Lesher A. Minimally invasive repair of pectus excavatum. J Vis Surg 2016;2:73.
2009;18:66-72.

24. Hebra A. Minimally invasive repair of pectus excavatum. Semin Thorac Cardiovasc Surg 2009;21:76-84.

25. Robicsek F, Hebra A. To Nuss or not to Nuss? Two opposing views. Semin Thorac Cardiovasc Surg. 2009;21:85-8. 